\title{
Erratum to "The Riemann Hypothesis-Millennium Prize Problem" [Advances in Pure Mathematics 6 (2016) 915-920]
}

\section{A. A. Durmagambetov}

L. N. Gumilyov Eurasian National University

Email: aset.durmagambet@gmail.com

How to cite this paper: Durmagambetov, A.A. (2016) Erratum to "The Riemann Hypothesis-Millennium Prize Problem" [Advances in Pure Mathematics 6 (2016) 915920]. Advances in Pure Mathematics, 6, 1053-1056.

http://dx.doi.org/10.4236/apm.2016.613077

Received: December 12, 2016

Accepted: December 25, 2016

Published: December 28, 2016

Copyright (๑) 2016 by author and Scientific Research Publishing Inc.

This work is licensed under the Creative

Commons Attribution International

License (CC BY 4.0).

http://creativecommons.org/licenses/by/4.0/
The original online version of this article (Durmagambetov, A.A. (2016) The Riemann Hypothesis-Millennium Prize Problem. Advances in Pure Mathematics, 6, 915-920. 10.4236/apm.2016.612069) unfortunately contains a mistake. The author wishes to correct the errors in Theorem 2 of the result part.

\section{Results}

These are the well-known Abel's results.

Theorem 1. Let the function $\phi(x)$ be limited on every finite interval, and $\frac{\mathrm{d} \phi}{\mathrm{d} x}(x)$ is continuous and limited on every finite interval then

$$
\sum_{a<n \leq b} \phi(n)=\int_{a}^{b} \phi(x) \mathrm{d} x+\int_{a}^{b}(x-[x]-1 / 2) \frac{\mathrm{d} \phi}{\mathrm{d} x} \mathrm{~d} x+(a-[a]-1 / 2) \phi(a)-(b-[b]-1 / 2) \phi(b)
$$

Corollary 1 . Let the function $s>1, \phi(x)=x^{-s}, a, b \in N$ then

$$
\begin{gathered}
\sum_{a<n \leq b} n^{-s}=\frac{b^{1-s}-a^{1-s}}{1-s}-s \int_{a}^{b} \frac{(x-[x]-1 / 2)}{x^{s+1}} \mathrm{~d} x+\frac{1}{2}\left(b^{-s}-a^{s}\right) \\
\sum_{1<n<\infty} n^{-s}=-\frac{1}{1-s}-s \int_{1}^{\infty} \frac{(x-[x]-1 / 2)}{x^{s+1}} \mathrm{~d} x+\frac{1}{2}\left(b^{-s}-a^{s}\right)
\end{gathered}
$$

Our goal is to use this theorem on the analogs of zeta functions. We are interested in the analytical properties of the following generalizations of zeta functions:

$$
\begin{gathered}
P(s)=\sum_{p} \frac{1}{p^{s}}, Q(s)=\sum_{p} \frac{1}{(p-1)^{s}} \\
P_{m}(s)=\sum_{p \leq m} \frac{1}{p^{s}}, Q_{m}(s)=\sum_{p \leq m} \frac{1}{(p-1)^{s}}
\end{gathered}
$$




$$
\begin{gathered}
P^{m}(s)=\sum_{p>m} \frac{1}{p^{s}}, Q^{m}(s)=\sum_{p>m} \frac{1}{(p-1)^{s}} \\
\varsigma_{p}^{m}(s)=\varsigma(s)-P^{m}(s)
\end{gathered}
$$

Let $N$ be the set of all natural numbers and $N_{p}^{m}=(n \in N, n \geq m, n$-prime number $)$ $N P_{m}=N / N_{p}^{m}$-the set of all natural numbers without $N_{p}^{m}$

Below we will always let $m>3$, this limitation is introduced only to simplify the calculations. Considering all the information above let us rewrite

$$
\varsigma_{p}^{m}(s)=\sum_{n \in N P_{m}} \frac{1}{n^{s}} .
$$

For the function $\varsigma_{p}^{m}(s)=\varsigma(s)-P^{m}(s)$, let us apply the results obtained by Muntz for the zeta function representation. With the help of the given definitions we formulate the analog of Muntz theorem.

Lemma 1. Let the function

$$
\begin{gathered}
\delta(s)=P^{m}(s)-Q^{m}(s), \text { then } \\
\delta(s)=-s P^{m}(s+1)+s^{2} O\left(P^{m}(s+2)\right) .
\end{gathered}
$$

PROOF: According to the theorem conditions we have

$$
\begin{aligned}
\delta(s) & =\sum_{p \in N_{p}^{m}}\left[\frac{1}{p^{s}}-\frac{1}{(p-1)^{s}}\right]=\sum_{p \in N_{p}^{m}} \frac{1}{p^{s}}\left[1-\frac{1}{(-1 / p+1)^{s}}\right] \\
& =-s \sum_{p \in N_{p}^{m}} \frac{1}{p^{s+1}}+s^{2} O\left(P^{m}(s+2)\right) .
\end{aligned}
$$

Lemma 2. Let the function

$$
\gamma 1(s)=\sum_{p \in N_{p}^{m}} \int_{p-1}^{p} \frac{x}{x^{s+1}} \mathrm{~d} x, \gamma 2(s)=-\sum_{p \in N_{p}^{m}} \int_{p-1}^{p} \frac{[x]}{x^{s+1}} \mathrm{~d} x, \gamma 3(s)=-\sum_{p \in N_{p}^{m}} \int_{p-1}^{p} \frac{1 / 2}{x^{s+1}} \mathrm{~d} x,
$$

then

$$
\begin{gathered}
\gamma 1(s)=\frac{1}{1-s} \sum_{p \in N_{p}^{m}}\left[\frac{1}{p^{s-1}}-\frac{1}{(p-1)^{s-1}}\right]=\frac{\delta(s-1)}{1-s} \\
\gamma 2(s)=-\frac{1}{s} \sum_{p \in N_{p}^{m}}\left[\frac{p-1}{p^{s}}-\frac{p-1}{(p-1)^{s}}\right]=-\frac{\delta(s-1)}{s}+\frac{P^{m}(s)}{s} \\
\gamma 3(s)=-\frac{1}{2 s} \sum_{p \in N_{p}^{m}}\left[\frac{1}{p^{s}}-\frac{1}{(p-1)^{s}}\right]=-\frac{\delta(s)}{2 s} . \\
s[\gamma 1(s)+\gamma 2(s)+\gamma 3(s)]=s\left[\frac{\delta(s-1)}{s-1}-\frac{\delta(s-1)}{s}+\frac{P^{m}(s)}{s}-\frac{\delta(s)}{2 s}\right]
\end{gathered}
$$

PROOF: Follows from computing of integrals.

Lemma 3. Let the function 


$$
\begin{gathered}
\phi(x)=x^{-s}, s>1, \quad a, b, m-\text { prime numbers } \\
(a, b) \bigcap N_{p}^{m}=\varnothing,\{a, a \geq m\}=N_{p}^{m} \text { then } \\
-\delta(s-1)-m^{1-s}=\sum_{a, b \in N_{p}^{m}}\left[(b-1)^{1-s}-a^{1-s}\right] \\
\sum_{a, b \in N_{p}^{m}} s \int_{a}^{b-1} \frac{(x-[x]-1 / 2)}{x^{s+1}} \mathrm{~d} x=s \int_{m}^{\infty} \frac{(x-(x)-1 / 2)}{x^{s+1}} \mathrm{~d} x-s[\gamma 1(s)+\gamma 2(s)+\gamma 3(s)] ;
\end{gathered}
$$

PROOF: Computing the sums, we have

$$
\sum_{a, b \in N_{p}^{m}}\left[(b-1)^{1-s}-a^{1-s}\right]=-m^{1-s}+\sum_{p \in N_{p}^{m}}\left[(p-1)^{1-s}-p^{1-s}\right]=-m^{1-s}-\delta(s-1)
$$

Theorem 2. Let the function

$$
\begin{gathered}
\phi(x)=x^{-s}, s>1, \quad a, b, m-\text { prime numbers } \\
(a, b) \bigcap N_{p}^{m}=\varnothing,\{a, a \geq m\}=N_{p}^{m} \text { then } \\
s P^{m}(s)=s(s)-\left[-m^{1-s}-s \int_{m}^{\infty} \frac{(x-[x]-1 / 2)}{x^{s+1}} \mathrm{~d} x-\delta(s)-m^{-s}+O\left(P^{m}(s+1)\right)\right]
\end{gathered}
$$

PROOF: Using Corollary 1. we have

$$
\begin{aligned}
& \varsigma_{p}^{m}(s)=\sum_{a, b \in N_{p}^{m}} \sum_{a<n<b} n^{-s} \\
& =\sum_{a, b \in N_{p}^{m}} \frac{(b-1)^{1-s}-a^{1-s}}{1-s}-\sum_{a, b \in N_{p}^{m}} s \int_{a}^{b-1} \frac{(x-[x]-1 / 2)}{x^{s+1}} \mathrm{~d} x \\
& +\frac{1}{2} \sum_{a, b \in N_{p}^{m}}\left((b-1)^{-s}-a^{-s}\right) \\
& \varsigma_{p}^{m}(s)=-\delta(s-1)-m^{1-s}-s \int_{m}^{\infty} \frac{(x-[x]-1 / 2)}{x^{s+1}} \mathrm{~d} x \\
& +s[\gamma 1(s)+\gamma 2(s)+\gamma 3(s)]-\delta(s)-m^{-s} \\
& =-\delta(s-1)-m^{1-s}-s \int_{m}^{\infty} \frac{(x-[x]-1 / 2)}{x^{s+1}} \mathrm{~d} x \\
& +s\left[\frac{\delta(s-1)}{s-1}-\frac{\delta(s-1)}{s}+\frac{P^{m}(s)}{s}-\frac{\delta(s)}{2 s}\right]-\delta(s)-m^{-s} \\
& \varsigma_{p}^{m}(s)=\delta(s-1)\left[\frac{s-2}{1-s}\right]+P^{m}(s)-m^{1-s}-s \int_{m}^{\infty} \frac{(x-[x]-1 / 2)}{x^{s+1}} \mathrm{~d} x-\delta(s)-m^{-s} \\
& \varsigma(s)-P^{m}=(s-2) P^{m}(s)+P^{m}(s)-m^{1-s}-s \int_{m}^{\infty} \frac{(x-[x]-1 / 2)}{x^{s+1}} \mathrm{~d} x \\
& -\delta(s)-m^{-s}+O\left(P^{m}(s+1)\right) \text {. } \\
& \varsigma(s)=s P^{m}(s)-m^{1-s}-s \int_{m}^{\infty} \frac{(x-[x]-1 / 2)}{x^{s+1}} d x-\delta(s)-m^{-s}+O\left(P^{m}(s+1)\right) .
\end{aligned}
$$




$$
s P^{m}(s)=\varsigma(s)-\left[-m^{1-s}-s \int_{m}^{\infty} \frac{(x-[x]-1 / 2)}{x^{s+1}} \mathrm{~d} x-\delta(s)-m^{-s}+O\left(P^{m}(s+1)\right)\right]
$$

From the last equation we obtain the regularity of the function $\varsigma_{p}^{m}(s), P^{m}(s)$ as $s$ satisfied $1 / 2<\operatorname{Re}(s)<1$.

Theorem 3. The Riemann's function has nontrivial zeros only on the line $\operatorname{Re}(s)=1 / 2$; PROOF: For $R 2(s)=\sum_{m=2}^{\infty} P(m s) / m$, we have

$$
|R 2(s)|=\left|\sum_{m=2}^{\infty} P(m s) / m\right| \leq \sum_{m=2}^{\infty}|P(m s) / m| \leq C_{\delta} \sum_{m=2}^{\infty}\left|-2^{m \delta} / m\right|<C C_{\delta}<\infty
$$

Applying the formula from the theorem 2

$$
\ln (\varsigma(s))=P(s)+\sum_{m=2}^{\infty} P(m s) / m=P(s)+R 2(s)=\varsigma(s)-\varsigma_{p}^{m}(s)-P_{m}(s)+R 2(s)
$$

estimating by the module

$$
\mid \ln \left(\varsigma(s)|\leq| \varsigma(s)|+| \varsigma_{p}^{m}(s)|+| R 2(s)|+| P_{m}(s) \mid .\right.
$$

Estimating the zeta function, potentiating, we obtain

$$
\mid\left(\varsigma((s)) \mid \geq \exp \left[-|\varsigma(s)|-\left|\varsigma_{p}^{m}(s)\right|-|R 2(s)|-\left|P_{m}(s)\right|\right]\right.
$$

According to the theorem $1|\varsigma(s)|$ limited for $\mathrm{z}$ from the following multitude

$$
(s,|s|<R,|s|>1+\delta, \delta>0)
$$

similarly, applying the theorem 2 for $\left|\varsigma_{p}^{m}(s)\right|$ we obtain its limitation in the same multitude. For the function $|R 2(s)|$ we have a limitation for all $\mathrm{z}$, belonging to the half-plane $\operatorname{Re}(s)>1 / 2+1 / \mathrm{R}$. similarly, applying the theorem 2 for $\left|\varsigma_{p}^{m}(s)\right|$ we obtain its limitation in the same multitude and finally we obtain:

$$
|(\varsigma(s))| \geq \exp \left[-C_{R}\right], \operatorname{Re}(s)>1 / 2+1 / R,|s|<R,|s|>1+\delta, \delta>0
$$

These estimations for $|P(s)|,|R 2(s)|,\left|P_{m}(s)\right|$ prove that zate function does not have zeros on the half-plane $\operatorname{Re}(s)>1 / 2+1 / R$ due to the integral representation (3) these results are projected on the half-plane $\operatorname{Re}(s)<1 / 2$ for the case of nontrivial zeros. The Riemann's hypothesis is proved. 